\title{
Study on demographic variables of controlled ovarian Hyperstimulation and Intrauterine Insemination (husband) in a tertiary medical college and hospital in Kolkata
}

\author{
Agniv Sarkar ${ }^{1}$, Rajib Roy ${ }^{2}$, Joydeb Roy Chowdhury ${ }^{3}$, Bibhas Saha Dalal ${ }^{4}$ \\ ${ }^{1}$ Post Graduate Trainee, ${ }^{2}$ Associate Professor, ${ }^{3}$ Dean and Director Professor, Department of Obstretics and Gynecology,
} ESI-PGIMSR, Joka, Kolkata, India, ${ }^{4}$ Assistant Professor, Department of Pathology, ESI-PGIMSR, Joka, Kolkata, India

Background: Intrauterine Insemination (IUI) is anage old reliable and safe procedure for couples having infertility. Success rate of IUI with ovulation induction ranges from $8-20 \%$ depending on many factors. Aims and Objectives: The current study was to assess the factors which are responsible for favourable outcome of IUI after ovulation induction. Materials and Methods: Total 67 couples were included fulfilling inclusion and exclusion criteria. They underwent 90 cycles of IUI with each couple having maximum of 3 cycles. Ovulation induction was done byeitherclomiphene citrate or letrozole or gonadotrophins. Semen preparation was done by density gradient method. The participant's demographic parameters were analyzed. Results: Out of $90 \mathrm{IUI}, 8$ were successful resulting in a success rate of $8.8 \%$ per cycle and $11.9 \%$ per couple. Factors which had positive impact were age less than 35 years, less than 10 years of infertility, normal and overweight BMI. Anovulation and unexplained infertility had better outcome. Type of infertility did not have any impact on the outcome. Conclusion: The study concluded that IUI after ovulation induction is more useful in cases like unexplained infertility and anovulation in a patient aged less than 35 years with less than 10 years of infertility and normal or overweight BMI.

Key words: BMI; Anovulation; Age; Demographic factors; Success rate

\section{Access this article online}

\section{Website:}

http://nepjol.info/index.php/AJMS DOI: 10.3126/ajms.v12i2.31270

E-ISSN: 2091-0576

P-ISSN: 2467-9100

Copyright (c) 2021 Asian Journal of Medical Sciences

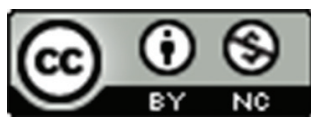

This work is licensed under a Creative Commons Attribution-NonCommercial 4.0 International License.

\section{INTRODUCTION}

Infertility is the inability to contribute to conception within 12 months of regular unprotected vaginal intercourse. Epidemiological data shows the prevalence to be $8-32 \% .{ }^{1,2}$ Causes can be divided into female, male, unexplained infertility. Female factors include ovulation disorder, tubal disease, endometriosis and all together accounts for 40 percent of total infertility. Male factors account for $30 \%$ and unexplained infertility $10 \% \cdot{ }^{3}$ Rest of them includes both male and female factors. A combination of controlled ovarian hyperstimulation or $\mathrm{COH}$ and intrauterine insemination or IUI remains an important option available to an infertility specialist and it is a widely used treatment modality for a broad range of indications. Common indications are cervical factor, anovulation, mild male factor, mild endometriosis, and unexplained infertility. These common conditions are well prevalent in the society and bear the bulk of the infertility cases both primary and secondary, especially with the changing socioeconomic characteristics in the society; the cases like anovulation due to PCOS and male factor infertility are on rise. In this regard IUI is a very simple, cost effective procedure but with limited success rate compared to IVF.

The reported pregnancy rates per cycle ranges from $8-22 \%{ }^{3}$ Commonly clomiphene is used as the drug of choice for ovulation induction, but recently letrozol is 
gaining more prominence and is on the verge of replacing clomiphene as the first line drug of choice for ovulation induction. Gonadotrophin is usually used in resistant cases and requires more vigilant monitoring of follicular growth. After ovulation induction the processed semen from either husband or donor is put into the uterine cavity through cervix via an insemination cannula. Several prognostic factors with regard to IUI outcome have been identified. It includes factors like patient profile, duration of infertility, type of infertility, stimulation protocol, follicular response, endometrial thickness, timing of IUI, semen parameters like post wash count. IUI is a simple low cost procedure without any significant side effects. Also in unexplained infertility it is a first line cost effective approach in developing countries rather than going for ART.

\section{AIMS AND OBJECTIVE}

To assess the demographic factors responsible for favourable outcome after ovulation induction and IUI.

\section{MATERIALS AND METHODS}

It is a prospective cross-sectional study done between a period of March 2018 to July 2019 in ESIC, Joka, Department of Gynecology and Department of Pathology. A total of 67 couples was identified through OPD andtotal 90 cycles of IUI were done in a period aforementioned. The study was pre-approved by the ethical committee of ESIC, Joka.

The inclusion criteria included females with unexplained infertility, endometriosis with at least one patent tube and only peritoneal spots $<5 \mathrm{~mm}$ depth found in laparoscopy (Mild endometriosis) and anovulation diagnosed by ultrasonography and mild male factor infertility (sperm count 5-15 million/ml).

Exclusion criteria were women with bilateral tubal block as seen by HSG followed by Diagnostic laparoscopy and Hysteroscopy, severe endometriosis (Endometriosis with bilateral tubal block or bilateral ovarian involvement), unexplained genital bleeding and pregnancy contraindicated due to various medical comorbidities like severe cardiac diseases. Also males with Severe male factor infertility (sperm count $<5$ million/ $\mathrm{ml}$ ) andPostwash sperm count $<5$ million/ $\mathrm{ml}$ were not included in the study.

Infertile couples were selected as per inclusion and exclusion criteria from OPD after taking their informed consent.A detailed history of both the partners were taken followed by general physical examination, differenthormonal tests like LH, FSH, Insulin, Thyroid function, Prolactin were carried out. Baseline antral follicle count, Follicular growth were monitored inunstimulated cycles to assess ovulation. BMI of the female patients were noted down and classified according to $\mathrm{WHO}$ [Normal $=18.5-24.9$, Overweight $=25-29.9$, Obesity gr $1=30-34.9$, Underweight $=<18.5]$. BMI of the males are also categorized in a similar fashion.

Once timed induction or hyperstimulation was decided the womenwere subjected to undergo Controlled ovarian hyperstimulation by eitherclomiphene citrate or purified FSH/HMG or letrozole in titrating doses from day 2 of menstrual cycle.After the hyperstimulation the patients were monitored by transvaginal sonography for follicular development from day 10 of menstrual cycle onwards. If one or more follicle size reaches more than $18 \mathrm{sq}$ $\mathrm{mm}$ and endometrial thickness reaches more than $7 \mathrm{~mm}$ and if there are no features of ovarian hyperstimulation syndrome (abdominal swelling, pain, vomiting, ascites) then the patient will be triggered with HCG injection $5000 \mathrm{U}$ or $10000 \mathrm{U}$ through intramuscular route for triggering the ovulation. The male partner was asked to attend within 36-42 hours after trigger for supplying the semen sample which was then processed by density gradient method.

The semen sample was obtained by masturbation after 72 hours of abstinence. At first a prewash count was taken with the help of neubers chamber and microscope. The media was preheated to $37^{\circ} \mathrm{C}$ in a thermal bath for 2 hours before the procedure starts. Then $1 \mathrm{ml}$ of $90 \%$ $(\mathrm{v} / \mathrm{v})$ media was taken in a conical tube (Falcon tube) with a sterile pipette. It was gently overlaid with $1 \mathrm{ml}$ of $45 \%$ $(\mathrm{v} / \mathrm{v})$ gradient media. The whole semen was then placed on top of two layers. It was centrifuged at $1500 \mathrm{rpm}$ for 15 minutes. The supernatant was discarded leaving the pellet with as little of media as possible. In another test tube $5 \mathrm{ml}$ of sperm wash media was taken and the pellet was transferred via pipette. It was then centrifuged at $1500 \mathrm{rpm}$ for 10 minutes. The supernatant was discarded and pellet with $0.5-1 \mathrm{ml}$ solution was prepared. A small amount of ( 0.2 micro liter) of this sample was put under microscope with Neubers chamber. Under 10X focal length the number of motile sperms were counted in the middle counting chamber of neubers chamber and if the post wash count was more than 5 million/ $\mathrm{ml}$ then the sample was taken for further steps. The final processed sample was used for insemination through insemination cannula. Urine pregnancy testwas done after 14 days and a transabdominal ultrasound was done after 6 weeks. Positive urine pregnancy test and ultrasonic evidence of pregnancy were taken as successful outcome. The 
participant's demographic parameters were analyzed. All the expenses were carried out by Department of Obstetrics and Gynecology, ESIC Joka. IUI set with insemination cannula supplied by Krischo Medical products Pvt Limited, Bangalore, Karnataka.

The statistical analysis was done by using p value $(<0.05)$ which will be statistically significant with $95 \%$ confidence interval. Data were entered in Microsoft Excel Sheet. Descriptive analysis by calculating percentages, confidence interval, mean with SD. Median and range. Appropriate statistical test was applied as per the need of study.

\section{RESULTS}

Total 90 IUI were done and out of 90 IUI 8 were successful. Total 67 couples underwent IUI and out of them 29 couple had 1 cycle of IUI, 23 couples had 2 cycles of IUI and 15 couple had 3 cycles. Common demographic factors we studied were age, duration of infertility, type of infertility, BMI of wife and causes of infertility. $\mathrm{p}$ value $<0.05$ were taken as statistically significant. Success rate were observed to be $20 \%$ below 25 years of age and $15.6 \%$ between 25 35 years [ Table 1]. Similarly it is $13.33 \%$ for infertility duration less than 5 years and $13.63 \%$ for duration between 5-10 years [Table 2]. The study also indicates that success rate is similar in both primary and secondary infertility [ $11.53 \%$ vs $13.33 \%$ ] [ Table 3]. It also shows in normal BMI [ 18.5-24.99] that of overweight BMI [ 25-29.99] successful conception occurs in $12.5 \%$ an $15 \%$ of cases respectively. [Table 4]. Finally the study also demonstrates that anovulation has highest success rate (16.6\%) followed by unexplained infertility (13.3\%).[ Table 5].

\section{DISCUSSION}

In this study above 35 years of age the success rate of IUI falls steeply to $4 \%$ which is statistically significant. The success rate is $15.62 \%$ between $25-35$ years of age and goes up to $20 \%$ below 25 years of age (Table 1 ).

Among the patient parameters female age is important as oocyte quality declines with increasing age. Success rate improves if the age is $<35$ years which is statistically significant. Age $>35$ years has a negative impact on success of IUI. It is probably due to a combination of progressive follicular depletion, decline in granulosa cell function, poor oocyte quality, reduced endometrial receptivity and higher rate of chromosomal abnormality. The rate is comparable to the result of study conducted elsewhere. ${ }^{4}$ Another study also observed that IUI success rate steeply reduces as age increases specially after 40 years. Similarly, one study reported $15.3 \%$ success rate for age $<25$ years which corroborates with our finding of $20 \%$ and gradually deteriorating with age. ${ }^{5}$

Duration of infertility is directly related to the success of IUI. In this study success rate is $13.46 \%$ for couples having infertility for less than 10 years whereas success rate downs by half to $6.67 \%$ in couples having infertility for more than 10 years (Table 2).Moreover, the success rate is similar between 5-10 years and less than 5 years. Though the results are in accordance with the fact that success rate reduces as the duration of infertility increases but the two other similar studies suggested that success rate reduces if duration is more than 5 years and further reduces after 10 years of infertility. ${ }^{6}$ Also the success rate in other studies found out to be $15.1 \%$ in duration less than 5 years which corroborates with our findings of $13.33 \%$ but it slightly increases in $5-10$ years as $13.63 \% .^{7}$

\begin{tabular}{lccc}
\multicolumn{4}{l}{ Table 1: Age wise success rate (pregnancy) } \\
\hline Age (years) & Success rate & Percentage (\%) & P value \\
\hline$<25$ & $2 / 10$ & 20 & 0.005 \\
$25-35$ & $5 / 32$ & 15.62 & 0.005 \\
$>35$ & $1 / 25$ & 4.00 & 0.006 \\
\hline
\end{tabular}

Table 2: Infertility duration wise success rate

\begin{tabular}{lccc}
\hline $\begin{array}{l}\text { Duration of } \\
\text { infertility (years) }\end{array}$ & $\begin{array}{c}\text { Success } \\
\text { rate }\end{array}$ & Percentage (\%) & p value \\
\hline$>5$ years & $4 / 30$ & 13.33 & 0.01 \\
$5-10$ years & $3 / 22$ & 13.63 & 0.01 \\
$>10$ years & $1 / 15$ & 6.67 & 0.5 \\
\hline
\end{tabular}

*IUI success rate doubles if duration of infertility is less than 10 years which is statistically significant

\begin{tabular}{lccc}
\multicolumn{4}{l}{ Table 3: Infertility type wise success rate } \\
\hline Type of infertility & Success rate & Percentage (\%) & P value \\
\hline Primary & $6 / 52$ & 11.53 & 0.18 \\
Secondary & $2 / 15$ & 13.33 & 0.2 \\
\hline
\end{tabular}

\begin{tabular}{lccc}
\multicolumn{4}{l}{ Table 4: Success rate by BMI of wife } \\
\hline BMI of wife & Success rate & Percentage (\%) & P value \\
\hline Normal & $5 / 40$ & 12.5 & 0.4 \\
Overweight & $3 / 20$ & 15 & 0.6 \\
Obesity gr1 & $0 / 3$ & 0 & 0.00 \\
Underweight & $0 / 4$ & 0 & 0.00 \\
\hline
\end{tabular}

\begin{tabular}{|c|c|c|c|}
\hline Causes & Success rate & Percentage (\%) & $P$ value \\
\hline Anovulation & $4 / 24$ & 16.66 & 0.02 \\
\hline Mild male factor & $2 / 23$ & 8.7 & 0.1 \\
\hline Unexplained & $2 / 15$ & 13.33 & 0.03 \\
\hline Mild endometriosis & $0 / 5$ & 00 & 0.00 \\
\hline
\end{tabular}

*Among the causes anovulation has highest success rate (16.6\%) followed by unexplained infertility (13.3\%). Both of these findings are statistically significant. 
This study demonstrates that success rate is similar in both primary and secondary infertility [ $11.53 \%$ vs $13.33 \%]$ (Table 3). The difference is not statistically significant. The result corresponds to findings of previous other studies. ${ }^{6,8}$ They did not find any statistically significant difference in the success rate by type of infertility in their study.

The study shows that success rate in normal BMI [18.524.99 ] is $12.5 \%$ and that of overweight BMI [25-29.99] is $15 \%$ but obesity grade 1 and underweight patients have poor success rate (Table 4). The findings are statistically not significant. However in another study it was also demonstrated that success rate in overweight female increases possibly because obesity is associated with PCOS, one of the commonest cause of anovulation leading to poor oocyte quality and oocyte depletion but also the findings suggest that anovulation is more commonly detected and treated and therefore success rate improves. ${ }^{7}$ Similar study conducted in 2018 found the success rate in normal BMI to be $15.1 \%$ which is similar to our finding of $12.5 \%$ but rate falls with increase in $\mathrm{BMI}$ in that study. ${ }^{7,9,10}$

Common causes which are included in this study are anovulation, mild male factor infertility, unexplained infertility, mild endometriosis. The success rate for anovulation is $16.66 \%$ and for unexplained infertility is $13.33 \%$ but the success rate drops down to $8.7 \%$ in mild male factor (Table 5). Therefore, IUI is more successful when the cause in anovulation and unexplained infertility and this result is statistically significant.It also corroborates with a previous study which showed success rate for unexplained infertility to be $15 \% .{ }^{11,12}$

\section{Limitations}

Sample size was small due to limited resources and limited time. Also due to the timeframe of one year all the 67 couples could not complete the entire 3 cycles of IUI. Furthermore 6 cycles of IUI would have given more success rate.

\section{CONCLUSION}

Success rate of IUI improves in the demographic scenario of female age less than 35 years, less than 10 years of infertility, normal and overweight BMI and in cases of anovulation and unexplained infertility. There is no relationship between success rate of IUI and types of infertility such as primary or secondary infertility. However further studies are required with larger sample size to validate the findings of this study.

\section{ACKNOWLEDGMENTS}

All the expenses are carried out by ESIC JOKA.The study has been approved by ethical clearance committee of ESIC, Joka.

\section{REFERENCES}

1. Ganguly I, Singh A and Bhandari S. Pregnancy predictors after intrauterineinsemination in cases of unexplained infertility: a prospective study. International journal of Reproductive Medicine. 2016; 5 pages.

https://doi.org/10.1155/2016/5817823

2. ESHRE: Focus on reproduction, The IUI revival in unexplained infertility, September 2017.

3. NICE: Fertility problems: assessment and treatment, February 2013.

4. Nuojua - HuttunenS, Tomas C, Blogiu R, Tuomivaara L and Martikainen $\mathrm{H}$. Intrauterine Insemination treatment in subfertility: an ananlysis of factors affecting outcome. Hum Reprod. 1999;14(3):698-703.

https://doi.org/10.1093/humrep/14.3.698

5. Vlahos NF, Coker I, Lawler C andZhao Y. Women with ovulatory dysfunction undergoing ovulation inductionwith clomiphene citrate vs administration of gonadotrophin.FertilSteril 2005; 83(5):1510-1516.

https://doi.org/10.1016/j.fertnstert.2004.11.049

6. Kamath MS, BhaveP and George K.Predictive factors for pregnancy after intrauterine insemination: A prospective study of factors affecting outcome. J Hum Reprod Sci. 2010; 3(3): 129-134. https://doi.org/10.4103/0974-1208.74154

7. Wadhwa L, Fauzdar A and Wadhwa SN. An intrauterine Insemination audit at tertiary care hospital: a 4 1/2 years retrospective analysis of 800 cycles. J Hum Reprod Sci. 2018; 11(3): 279-285.

https://doi.org/10.4103/jhrs.JHRS_34_18

8. Marcio Soria MI, Pradillo G and Garcia J. Pregnancy predictor after IUI: Analysis of 3012 cycles in 1201 couples. J Reprod Infertil 2012; 13(3): 158-166.

9. Li S, He Y, CaoM, Liu H and Liu J. Low dose human menopausal gonadotrophin versus natural cycles in intrauterine insemination for subfertile couples with regular menstruation. J Ovarian Res.2020;13(1):36.

https://doi.org/10.1186/s13048-020-00638-3

10. Gubert PG, Pudwell J, Van Vugt D, Reid RL and Velez MP. Number of motile spermatozoa inseminated and pregnancy outcomes in intrauterine insemination. Fertil Res and Pract.2019;5:10. https://doi.org/10.1186/s40738-019-0062-z

11. Hughes EG. The effectiveness of ovulation induction and intrauterine insemination in the treatment of persistent infertility; a meta analysis. Hum Reprod. 1997; 12(9): 1865-1872.

https://doi.org/10.1093/humrep/12.9.1865

12. Dickey RP, Taylor SN, Lu Py, Sartor BM, Rye PH andPyrzakR. The Fertility Institute of New Orleans, New Orleans,Louisiana [effect of diagnosis, age, sperm quality and number of preovulatory follicles on the outcome of multiple cycles of clomiphene citrate -intrauterine insemination]. Fertilsteril. 2002; 78(5): 1088-1095

https://doi.org/10.1016/S0015-0282(02)04212-7 
Author's Contribution:

AS- Design of the study, Preparation of first draft, Manuscript preparation; RR- Concept of the study, Review of literature, Critical revision of manuscript;

JRC- Concept and contribution of overall study; BSD- Statistical analysis and interpretation, review of literature.

Work attributed to:

Department of Obstretics and Gynaecology, ESI-PGIMSR, Joka, Kolkata.

Orcid ID:

Dr. Rajib Roy - (D) https://orcid.org/0000-0001-7342-3336

Source of Support: ESI-PGIMSR, JOKA, Conflict of Interest: None declared. 